\title{
Highly Enantioselective Conjugate Addition of Nitromethane to Chalcones Using Bifunctional Cinchona Organocatalysts
}

\author{
Benedek Vakulya, ${ }^{a}$ Szilárd Varga, ${ }^{a}$ Antal Csámpai ${ }^{b}$ and Tibor Soós* ${ }^{* a}$ \\ Supporting Information \\ ${ }^{a}$ Institute of Biomolecular Chemistry, Chemical Research Center of Hungarian Academy of Sciences, Budapest, \\ Hungary P.O. Box 17, 1525 \\ ${ }^{b}$ Department of General and Inorganic Chemistry, Eötvös Loránd University, Budapest, Hungary P.O. Box 32, 1518
}

General. Nuclear Magnetic Resonance spectra (NMR) were acquired on a Bruker DRX-500 (500 MHz) instrument using TMS or $\mathrm{NH}_{3}$ as internal standard. ${ }^{15} \mathrm{~N}-\mathrm{NMR}$ chemical shifts of the potential cinchona catalysts were obtained by ${ }^{1} \mathrm{H}-{ }^{15} \mathrm{~N}-\mathrm{HMBC}$ experiments. All assignments are confirmed by 2D-COSY, 2D-HSC, 2D-HMBC and DNOE measurements. IR spectra were recorded on a Nicolet Avatar 320 FT-IR spectrophotometer and are reported in wavenumbers $\left(\mathrm{cm}^{-1}\right)$. Exact mass (HR-MS) spectra were recorded on a VG ZAB2-SEQ tandem mass spectrometer. Elemental analyses were performed with a Fisons EA 1108 instrument. For column cromatography, Merck Silica gel 60 and for flash cromatography Merck Silica gel 60H were employed. Enantiomer ratios were determined by chiral HPLC analysis using a Waters 600 and Waters 996 Photodiode Array Detector with a Chiralpak AD column $(0.46 \mathrm{~cm} \times 25 \mathrm{~cm})$.

Materials. Hydroquinine, quinine, quinidine, and diisopropyl azodicarboxylate were purchased from Fluka. Diphenylphosphoryl azide and trans-chalcone were purchased from Aldrich Inc. Substituted chalcones ${ }^{1-d}$ and amine substituted epiquinine ${ }^{2}$, epiquinidine ${ }^{3}$ and quinine ${ }^{4}$ were prepared as described in literature. THF was distilled from sodium/benzophenone prior to use.

9-amino(9-deoxy)epihydroquinine (S1). Hydroquinine $\quad\left(\begin{array}{lllll}3.26 & \mathrm{~g}, & 10.0 & \mathrm{mmol}\end{array}\right)$ and triphenylphosphine $(3.15 \mathrm{~g}, 12.0 \mathrm{mmol})$ were dissolved in $50 \mathrm{~mL}$ of dry THF and the solution was 
cooled to $0{ }^{\circ} \mathrm{C}$. Diisopropyl azodicarboxylate $(2.43 \mathrm{~g}, 12.0 \mathrm{mmol})$ was added all at once. Then solution of diphenyl phosphoryl azide $(3.30 \mathrm{~g}, 12.0 \mathrm{mmol})$ in $20 \mathrm{~mL}$ of dry THF was added dropwise at $0{ }^{\circ} \mathrm{C}$. The mixture was allowed to warm to room temperature. After being stirred for 12 $\mathrm{h}$, the solution was heated to $50{ }^{\circ} \mathrm{C}$ for $2 \mathrm{~h}$. Then triphenylphosphine $(3.41 \mathrm{~g}, 13.0 \mathrm{mmol})$ was added and heating was maintained until the gas evolution has ceased $(2 \mathrm{~h})$. The solution was cooled to room temperature, and $1 \mathrm{~mL}$ of water was added and the solution was stirred for $3 \mathrm{~h}$. Solvents were removed in vacuo and the residue was dissolved in $\mathrm{CH}_{2} \mathrm{Cl}_{2}$ and $10 \%$ hydrochloric acid (1:1, $100 \mathrm{~mL})$. The aqueous phase was washed with $\mathrm{CH}_{2} \mathrm{Cl}_{2}(4 \times 50 \mathrm{~mL})$. Then the aqueous phase was made alkaline with excess cc. aqueous ammonia and was washed with $\mathrm{CH}_{2} \mathrm{Cl}_{2}(4 \times 50 \mathrm{~mL})$. The combined organic phases was dried over $\mathrm{Na}_{2} \mathrm{SO}_{4}$ and concentrated. The residue was purified by column chromatography on silica gel (EtOAc/ $\mathrm{MeOH} / \mathrm{cc}$. aq. $\mathrm{NH}_{4} \mathrm{OH}=50 / 50 / 1$ as eluant) affording the title compound as a yellowish viscuous oil. $(2.30 \mathrm{~g}, 71 \%)$ : $[\alpha]_{\mathrm{D}}{ }^{23}+71.8\left(c 0.97, \mathrm{CHCl}_{3}\right)$;

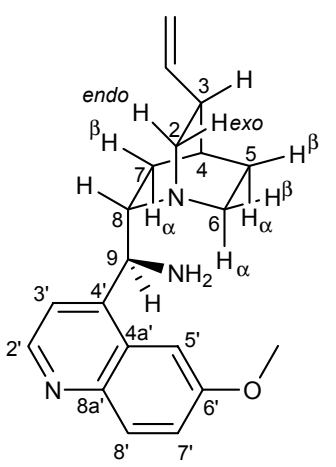

${ }^{1} \mathrm{H}-\mathrm{NMR}\left(500 \mathrm{MHz}, \mathrm{CD}_{3} \mathrm{OD}, \mathrm{T}=310 \mathrm{~K}, \delta_{\mathrm{TMS}}=0 \mathrm{ppm}\right) \delta 8.69(\mathrm{~d}, J=4.7$ Hz, 1H, H-2'), 7.97 (d, $J=9.3$ Hz, 1H, H-8'), 7.69 (br s, 1H, H-5'), 7.61 (d, $J$ $\left.=4.7 \mathrm{~Hz}, 1 \mathrm{H}, \mathrm{H}-3^{\prime}\right), 7.45\left(\mathrm{dd}, J=9.3,2.6 \mathrm{~Hz}, 1 \mathrm{H}, \mathrm{H}-7^{\prime}\right), 4.72(\mathrm{~d}, J=11.0$ $\mathrm{Hz}, 1 \mathrm{H}, \mathrm{H}-9), 4.00$ (s, 3H, $\mathrm{OCH}_{3}$ ), 3.32 (dddd, $J=15.6,10.5,7.8,2.3 \mathrm{~Hz}$, 1H, H-6 $\alpha$ ), 3.28 (dd, $J=13.6,9.9 \mathrm{~Hz}, 1 \mathrm{H}, \mathrm{H}-2$-exo), 3.16 (pseudo q, $J=10.7$ Hz, 1H, H-8), 2.79 (ddd, $J=15.6,13.8,4.9 \mathrm{~Hz}, 1 \mathrm{H}, \mathrm{H}-6 \beta$ ), 2.56 (ddd, $J=$ 13.6, 4.7, $2.3 \mathrm{~Hz}, 1 \mathrm{H}, \mathrm{H}-2$-endo), 1.60 (unresolved partly overlapping signal,

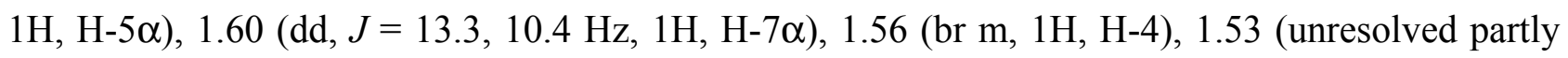
overlapping signal, $1 \mathrm{H}, \mathrm{H}-5 \beta$ ), 1.53 (ddd, $J=13.3,10.4,2.7 \mathrm{~Hz}, 1 \mathrm{H}, \mathrm{H}-7 \beta$ ), 1.47 (br m, 1H, H-3), $1.35\left(\mathrm{~m}, 2 \mathrm{H}, \mathrm{CH}_{2} \mathrm{CH}_{3}\right), 0.85$ (t, $\left.J=7.3 \mathrm{~Hz}, 3 \mathrm{H}, \mathrm{CH}_{2} \mathrm{CH}_{3}\right) \mathrm{ppm} ;{ }^{13} \mathrm{C}-\mathrm{NMR}\left(125 \mathrm{MHz}, \mathrm{CD}_{3} \mathrm{OD}, \mathrm{T}=\right.$ $310 \mathrm{~K}, \delta_{\text {TMS }}=0$ ppm) $\delta 158.8\left(\mathrm{C}-6^{\prime}\right), 148.3\left(\mathrm{C}^{\prime} 4^{\prime}\right), 147.5$ (C-2'), $144.2\left(\mathrm{C}-8 \mathrm{a}^{\prime}\right), 130.6$ (C-8'), 129.4 (C-4a'), 122.3 (C-7'), 120.2 (C-3'), 102.1 (C-5'), 62.2 (C-8), 57.8 (C-2), $55.2\left(\mathrm{OCH}_{3}\right), 51.9$ (C-9), 40.8 (C-6), 37.8 (C-3), $28.6(\mathrm{C}-5), 27.6\left(\mathrm{CH}_{2} \mathrm{CH}_{3}\right), 25.8(\mathrm{C}-7), 25.7(\mathrm{C}-4), 11.4\left(\mathrm{CH}_{2} \mathrm{CH}_{3}\right)$ ppm; ${ }^{15} \mathrm{~N}-\mathrm{NMR}\left(50 \mathrm{MHz}, \mathrm{CD}_{3} \mathrm{OD}, \mathrm{T}=310 \mathrm{~K}, \delta_{\mathrm{NH}_{3}}=0 \mathrm{ppm}\right) \delta 25$ (quinuclidine-N), 293 (quinoline-N), $\mathrm{NH}_{2}$ could not be detected under the applied conditions. IR $\left(\mathrm{CH}_{2} \mathrm{Cl}_{2}\right) \vee 1622,1507,1473,1433$, 1265, 1032, 915, 737, $635 \mathrm{~cm}^{-1}$; HRMS (EI) Exact mass calculated for $\mathrm{C}_{20} \mathrm{H}_{27} \mathrm{~N}_{3} \mathrm{O}$ [M] 325.2154; 
Found: 325.2160. Anal. Calcd. for $\mathrm{C}_{20} \mathrm{H}_{27} \mathrm{~N}_{3} \mathrm{O}$ : C, 73.81; H, 8.36; N, 12.91; O, 4.92. Found: $\mathrm{C}$, 73.75; H, 8.33; N, 12.75.

\section{General Procedure for Preparing Thiourea Catalysts. Reaction of 9-amino(9- deoxy)epihydroquinine (S1) is typical.}

To a solution of 9-amino(9-deoxy)epihydroquinine (S1, $2.20 \mathrm{~g}, 6.8 \mathrm{mmol})$ in dry THF $(20 \mathrm{~mL})$ was slowly added a solution of 3,5-bis(trifluoromethyl)phenyl isothiocyanate $(1.84 \mathrm{~g}, 6.8 \mathrm{mmol})$ in 10 $\mathrm{mL}$ of dry THF at ambient temperature. The mixture was stirred overnight, and the solvent was removed in vacuo. The residue was purified by column chromatography on silica gel $\left(\mathrm{EtOAc} / \mathrm{MeOH} / \mathrm{cc}\right.$. aq. $\mathrm{NH}_{4} \mathrm{OH}=300 / 5 / 1$ as eluant) affording thiourea $\mathbf{5 b}(3.30 \mathrm{~g}, 81 \%)$ as an offwhite amorphous solid.

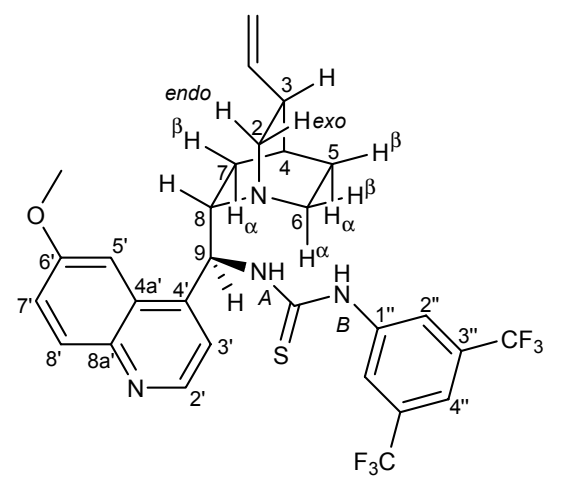

5a. Amorphous solid. Yield: $88 \% .[\alpha]_{\mathrm{D}}^{25}-127.9$ (c 0.50 , $\left.\mathrm{CHCl}_{3}\right) ;{ }^{1} \mathrm{H}-\mathrm{NMR}\left(500 \mathrm{MHz}, \mathrm{CD}_{3} \mathrm{OD}, \mathrm{T}=310 \mathrm{~K}, \delta_{\mathrm{TMS}}=0\right.$ ppm) $\delta 8.68$ (d, $J=4.7 \mathrm{~Hz}, 1 \mathrm{H}, \mathrm{H}-2$ '), 8.11 (br s, 2H, H-2'), 8.07 (br d, $J=2.6 \mathrm{~Hz}, 1 \mathrm{H}, \mathrm{H}-5$ ') 7.95 (d, $J=9.3 \mathrm{~Hz}, 1 \mathrm{H}, \mathrm{H}-8^{\prime}$ ), 7.59 (br s, 1H, H-4'), 7.55 (d, $J=4.7 \mathrm{~Hz}, 1 \mathrm{H}, \mathrm{H}-3$ '), 7.44 (dd, $J$ = 9.3, $\left.2.6 \mathrm{~Hz}, 1 \mathrm{H}, \mathrm{H}^{-} 7^{\prime}\right), 6.32(\mathrm{~d}, J=11.0 \mathrm{~Hz}, 1 \mathrm{H}, \mathrm{H}-9), 5.84$ (ddd, $\left.J=17.2,10.5,6.2 \mathrm{~Hz}, 1 \mathrm{H}, \mathrm{CH}=\mathrm{CH}_{2}\right), 5.02(\mathrm{dt}, J=10.5$, $\left.1.5 \mathrm{~Hz}, 1 \mathrm{H}, \mathrm{CH}=\mathrm{CH}_{2}\right), 4.98\left(\mathrm{dt}, J=17.2,1.5 \mathrm{~Hz}, 1 \mathrm{H}, \mathrm{CH}=\mathrm{CH}_{2}\right), 4.03\left(\mathrm{~s}, 3 \mathrm{H}, \mathrm{OCH}_{3}\right), 3.56$ (dddd, $J$ $=15.6,10.5,7.8,2.3 \mathrm{~Hz}, 1 \mathrm{H}, \mathrm{H}-6 \alpha$ ), 3.39 (br pseudo q, $J=10.7 \mathrm{~Hz}, 1 \mathrm{H}, \mathrm{H}-8$ ), 3.29 (dd, $J=13.6$, $9.9 \mathrm{~Hz}, 1 \mathrm{H}, \mathrm{H}-2$-exo), 2.82 (ddd, $J=15.6,13.8,4.9 \mathrm{~Hz}, 1 \mathrm{H}, \mathrm{H}-6 \beta$ ), 2.79 (ddd, $J=13.6,4.7,2.3$ $\mathrm{Hz}, 1 \mathrm{H}, \mathrm{H}-2$-endo), 2.36 (br m, 1H, H-3), 1.70 (overlapping br m, 2H, H-5 $\alpha$ and $\mathrm{H}-5 \beta$ ), 1.63 (br m, 1H, H-4), 1.45 (ddd, $J=13.3,10.4,2.7 \mathrm{~Hz}, 1 \mathrm{H}, \mathrm{H}-7 \beta$ ), 0.89 (dd, $J=13.3,10.4 \mathrm{~Hz}, 1 \mathrm{H}, \mathrm{H}-7 \alpha$ ) ppm; ${ }^{13} \mathrm{C}-\mathrm{NMR}\left(125 \mathrm{MHz}, \mathrm{CD}_{3} \mathrm{OD}, \mathrm{T}=310 \mathrm{~K}, \delta_{\mathrm{TMS}}=0 \mathrm{ppm}\right) \delta 181.6(\mathrm{C}=\mathrm{S}), 158.7$ (C-6'), $147.3\left(\mathrm{C}-2^{\prime}\right)$, 146.6 (C-4'), 144.2 (C-8a'), 142.0 (C-1' '), $141.5\left(\mathrm{CH}=\mathrm{CH}_{2}\right), 131.8$ (q, ${ }^{2} J_{\mathrm{CF}}=33.0 \mathrm{~Hz}, \mathrm{C}-3$ ' '), 130.3 (C-8'), 129.2 (C-4a'), 123.6 (q, ${ }^{1} J_{\mathrm{CF}}=272.2 \mathrm{~Hz}, \mathrm{CF}_{3}$ ), 122.7 (C-7'), 122.6 (C-2'), 120.2 (C-3'), 116.9 (sept, ${ }^{3} J_{\mathrm{CF}}=3.7 \mathrm{~Hz}, \mathrm{C}-4$ ") $, 114.0\left(\mathrm{CH}=\mathrm{CH}_{2}\right), 103.3(\mathrm{C}-5$ '), $60.7(\mathrm{C}-8), 55.8(\mathrm{C}-2), 55.5$ $\left(\mathrm{OCH}_{3}\right), 55.4$ (C-9), 41.8 (C-6), 39.7 (C-3), 27.8 (C-4), 27.7 (C-5), 25.9 (C-7) ppm; ${ }^{15} \mathrm{~N}-\mathrm{NMR}(50$ 
$\left.\mathrm{MHz}, \mathrm{CD}_{3} \mathrm{OD}, \mathrm{T}=310 \mathrm{~K}, \delta_{\mathrm{NH}_{3}}=0 \mathrm{ppm}\right) \delta 25$ (quinuclidine-N), 294 (quinoline-N), $131\left(\mathrm{~N}_{\mathrm{A}}\right), 125$ $\left(\mathrm{N}_{\mathrm{B}}\right) \mathrm{ppm}$; IR $(\mathrm{KBr}) \vee 1623,1510,1474,1384,1278,1179,1133,850,683 \mathrm{~cm}^{-1}$; HRMS (EI) Exact mass calculated for $\mathrm{C}_{29} \mathrm{H}_{28} \mathrm{~F}_{6} \mathrm{~N}_{4} \mathrm{OS}[\mathrm{M}]^{+}$594.1888; Found:

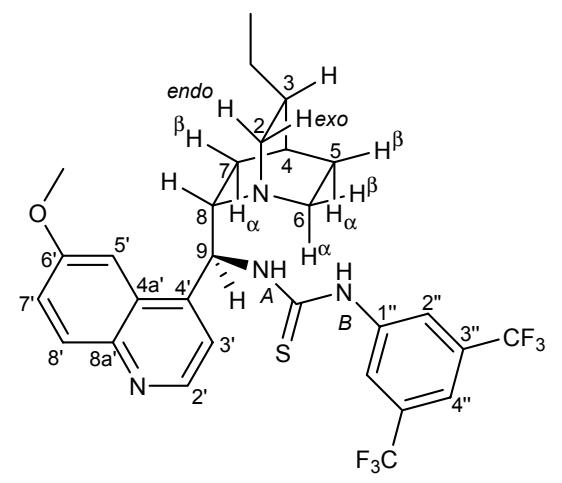
594.1878.

5b Amorphous solid. Yield: $81 \% . \quad[\alpha]_{\mathrm{D}}^{25}-124.6(c) 0.50$, $\left.\mathrm{CHCl}_{3}\right) ;{ }^{1} \mathrm{H}-\mathrm{NMR}\left(500 \mathrm{MHz}, \mathrm{CD}_{3} \mathrm{OD}, \mathrm{T}=310 \mathrm{~K}, \delta_{\mathrm{TMS}}=0 \mathrm{ppm}\right)$ $\delta 8.69$ (d, $J=4.7 \mathrm{~Hz}, 1 \mathrm{H}, \mathrm{H}-2$ '), 8.11 (br s, 2H, H-2'), 8.08 (br d, $J=2.6 \mathrm{~Hz}, 1 \mathrm{H}, \mathrm{H}-5^{\prime}$ ), 7.96 (d, $J=9.3 \mathrm{~Hz}, 1 \mathrm{H}, \mathrm{H}-8^{\prime}$ ), 7.59 (br s, 1H, H-4'), 7.57 (d, $J=4.7 \mathrm{~Hz}, 1 \mathrm{H}, \mathrm{H}-3$ '), 7.44 (dd, $J=9.3$, $2.6 \mathrm{~Hz}, 1 \mathrm{H}, \mathrm{H}-7$ ') 6.33 (d, $J=11.0 \mathrm{~Hz}, 1 \mathrm{H}, \mathrm{H}-9$ ), 4.03 (s, 3H, $\mathrm{OCH}_{3}$ ), 3.57 (dddd, $J=15.6,10.5$, 7.8, $2.3 \mathrm{~Hz}, 1 \mathrm{H}, \mathrm{H}-6 \alpha$ ), 3.37 (pseudo q, $J=10.7 \mathrm{~Hz}, 1 \mathrm{H}, \mathrm{H}-8$ ), 3.29 (dd, $J=13.6,9.9 \mathrm{~Hz}, 1 \mathrm{H}, \mathrm{H}-2-$ exo), 2.82 (ddd, $J=15.6,13.8,4.9 \mathrm{~Hz}, 1 \mathrm{H}, \mathrm{H}-6 \beta$ ), 2.53 (ddd, $J=13.6,4.7,2.3 \mathrm{~Hz}, 1 \mathrm{H}, \mathrm{H}-2$-endo), 1.70 (overlapping br m, 2H, H-5 $\alpha$ and $\mathrm{H}-5 \beta$ ), 1.53 (br m, 1H, H-3), 1.40 (br m, 1H, H-4), 1.38 (ddd, $J=13.3,10.4,2.7 \mathrm{~Hz}, 1 \mathrm{H}, \mathrm{H}-7 \beta), 1.32\left(\mathrm{~m}, 2 \mathrm{H}, \mathrm{CH}_{2} \mathrm{CH}_{3}\right), 0.86\left(\mathrm{t}, J=7.3 \mathrm{~Hz}, 3 \mathrm{H}, \mathrm{CH}_{2} \mathrm{CH}_{3}\right.$ ), $0.70(\mathrm{dd}, J=13.3,10.4 \mathrm{~Hz}, 1 \mathrm{H}, \mathrm{H}-7 \alpha)$, ppm; ${ }^{13} \mathrm{C}-\mathrm{NMR}\left(125 \mathrm{MHz}, \mathrm{CD}_{3} \mathrm{OD}, \mathrm{T}=310 \mathrm{~K}, \delta_{\mathrm{TMS}}=0\right.$ ppm) $\delta 181.6$ (C=S), 158.7 (C-6'), 147.3 (C-2'), 146.7 (C-4'), 144.2 (C-8a'), 142.1 (C-1'”), 131.8 (q, ${ }^{2} J_{\mathrm{CF}}=33.0 \mathrm{~Hz}, \mathrm{C}-3^{\prime}$ ') $, 130.3\left(\mathrm{C}-8^{\prime}\right), 129.2\left(\mathrm{C}-4 \mathrm{a}^{\prime}\right), 123.6\left(\mathrm{q},{ }^{1} J_{\mathrm{CF}}=272.2 \mathrm{~Hz}, \mathrm{CF}_{3}\right), 122.7$ (coalesced lines, C-2"' and C-7'), 120.2 (C-3'), 116.9 (br signal, ${ }^{3} J_{\mathrm{CF}}=3.7 \mathrm{~Hz}, \mathrm{C}-4$ '”), 103.3 (C-5'), 60.6 (C-8), 57.5 (C-2), $55.5\left(\mathrm{OCH}_{3}\right), 55.3(\mathrm{C}-9), 41.9(\mathrm{C}-6), 37.4(\mathrm{C}-3), 28.3(\mathrm{C}-5), 27.4\left(\mathrm{CH}_{2} \mathrm{CH}_{3}\right)$, 25.7 (C-7), 25.5 (C-4), $11.5\left(\mathrm{CH}_{2} \mathrm{CH}_{3}\right) \mathrm{ppm} ;{ }^{15} \mathrm{~N}-\mathrm{NMR}\left(50 \mathrm{MHz}, \mathrm{CD}_{3} \mathrm{OD}, \mathrm{T}=310 \mathrm{~K}, \delta_{\mathrm{NH}_{3}}=0\right.$ ppm) $\delta 25$ (quinuclidine-N), 294 (quinoline-N), $131\left(\mathrm{~N}_{\mathrm{A}}\right), 125\left(\mathrm{~N}_{\mathrm{B}}\right) \mathrm{ppm}$; IR $(\mathrm{KBr}) \vee$ 1623, 1510, $1474,1385,1278,1181,1135,681 \mathrm{~cm}^{-1}$; HRMS (EI) Exact mass calculated for $\mathrm{C}_{29} \mathrm{H}_{30} \mathrm{~F}_{6} \mathrm{~N}_{4} \mathrm{OS}[\mathrm{M}]^{+}$ 596.2045; Found: 596.2038. 


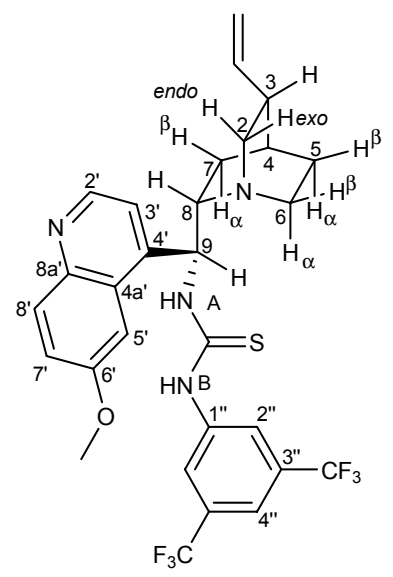

6. Amorphous solid. Yield: $67 \%$. $[\alpha]_{\mathrm{D}}{ }^{25}+28.6\left(c 0.50, \mathrm{CHCl}_{3}\right) ;{ }^{1} \mathrm{H}-$ NMR $\left(500 \mathrm{MHz}, \mathrm{CD}_{3} \mathrm{OD}, \mathrm{T}=310 \mathrm{~K}, \delta_{\mathrm{TMS}}=0 \mathrm{ppm}\right) \delta 8.70(\mathrm{~d}, J=4.7 \mathrm{~Hz}$, 1H, H-2'), 8.16 (br d, $J=2.6$ Hz, 1H, H-5'), 8.00 (br s, 2H, 2H-2'’), 7.95 (d, $J=9.3 \mathrm{~Hz}, 1 \mathrm{H}, \mathrm{H}-8^{\prime}$ ), 7.61 (br s, 1H, H-4’'), 7.58 (d, $J=4.7 \mathrm{~Hz}, 1 \mathrm{H}$, H-3'), 7.42 (dd, $J=9.3,2.6$ Hz, 1H, H-7'), 6.83 (d, $J=11.0$ Hz, 1H, H-9), $6.04\left(\mathrm{ddd}, J=17.2,10.5,6.2 \mathrm{~Hz}, 1 \mathrm{H}, \mathrm{CH}=\mathrm{CH}_{2}\right), 5.12(\mathrm{dt}, J=10.5,1.5 \mathrm{~Hz}$, $\left.1 \mathrm{H}, \mathrm{CH}=\mathrm{CH}_{2}\right), 5.09\left(\mathrm{dt}, J=17.2,1.5 \mathrm{~Hz}, 1 \mathrm{H}, \mathrm{CH}=\mathrm{CH}_{2}\right), 4.02(\mathrm{~s}, 3 \mathrm{H}$, $\mathrm{OCH}_{3}$ ), 3.69 (br pseudo q, $J=10.7 \mathrm{~Hz}, 1 \mathrm{H}, \mathrm{H}-8$ ), 3.13 (dddd, $J=15.6$, 10.5, 7.8, $2.3 \mathrm{~Hz}, 1 \mathrm{H}, \mathrm{H}-6 \alpha$ ), 3.02 (dd, $J=13.6,9.9 \mathrm{~Hz}, 1 \mathrm{H}, \mathrm{H}-2-$-exo), 2.71 (ddd, $J=13.6,4.7,2.3 \mathrm{~Hz}, 1 \mathrm{H}, \mathrm{H}-2$-endo), 2.57 (ddd, $J=15.6,13.8,4.9 \mathrm{~Hz}, 1 \mathrm{H}, \mathrm{H}-6 \beta), 2.35$ (br m, 1H, H-3), 2.09 (closely coupled m, 1H, H-7 $\beta$ ), 2.05 (closely coupled m, 1H, H-7 $\alpha$ ), 1.96 (unresolved partly overlapping signal, $1 \mathrm{H}, \mathrm{H}-5 \alpha$ ), 1.88 (pseudo sext, $J=3.3 \mathrm{~Hz}, 1 \mathrm{H}, \mathrm{H}-4$ ), 1.60 (unresolved partly overlapping signal, $1 \mathrm{H}, \mathrm{H}-5 \beta) \mathrm{ppm} ;{ }^{13} \mathrm{C}-\mathrm{NMR}\left(125 \mathrm{MHz}, \mathrm{CD}_{3} \mathrm{OD}, \mathrm{T}=310 \mathrm{~K}\right.$, $\left.\delta_{\mathrm{TMS}}=0 \mathrm{ppm}\right) \delta 182.6(\mathrm{C}=\mathrm{S}), 158.9$ (C-6’), 147.4 (C-2'), 146.4 (C-4'), 144.5 (C-8a'), 141.9 $\left(C \mathrm{H}=\mathrm{CH}_{2}\right), 141.5\left(\mathrm{C}-1^{\prime \prime}\right), 131.9$ (q, ${ }^{2} J_{\mathrm{CF}}=33.0 \mathrm{~Hz}, \mathrm{C}-3$ '’), 130.3 (C-8'), 129.1 (C-4a'), 123.6 (q, ${ }^{1} J_{\mathrm{CF}}=272.2 \mathrm{~Hz}, \mathrm{CF}_{3}$ ), 123.0 (C-2'’), 122.9 (C-7'), 119.7 (C-3'), 117.2 (br signal, ${ }^{3} J_{\mathrm{CF}}=3.7 \mathrm{~Hz}, \mathrm{C}-$ 4'’), $114.0\left(\mathrm{CH}=\mathrm{CH}_{2}\right), 103.2\left(\mathrm{C}-5\right.$ ') $59.1(\mathrm{C}-8), 56.0\left(\mathrm{OCH}_{3}\right), 55.7$ (C-2), 54.2 (C-9), 41.8 (C-6), 39.8 (C-3), 28.0 (C-4), 27.3 (C-5), 24.4 (C-7) ppm; ${ }^{15} \mathrm{~N}-\mathrm{NMR}\left(50 \mathrm{MHz}, \mathrm{CD}_{3} \mathrm{OD}, \mathrm{T}=310 \mathrm{~K}, \delta_{\mathrm{NH}_{3}}=\right.$ 0 ppm) $\delta 27$ (quinuclidine-N), 293 (quinoline-N), $128\left(\mathrm{~N}_{\mathrm{A}}\right), 124\left(\mathrm{~N}_{\mathrm{B}}\right) \mathrm{ppm}$; IR $(\mathrm{KBr}) \mathrm{v} 1623,1542$, 1513, 1474, 1385, 1278, 1178, 1134, 955, 847, $681 \mathrm{~cm}^{-1}$; MS (FAB) Mass calculated for $\mathrm{C}_{29} \mathrm{H}_{28} \mathrm{~F}_{6} \mathrm{~N}_{4} \mathrm{OS}[\mathrm{M}+\mathrm{H}]^{+}$595; Found: 595; HRMS (EI) Exact mass calculated for $\mathrm{C}_{29} \mathrm{H}_{28} \mathrm{~F}_{5} \mathrm{~N}_{4} \mathrm{OS}$ [MF] $]^{+}$575.1904; Found: 575.1892.

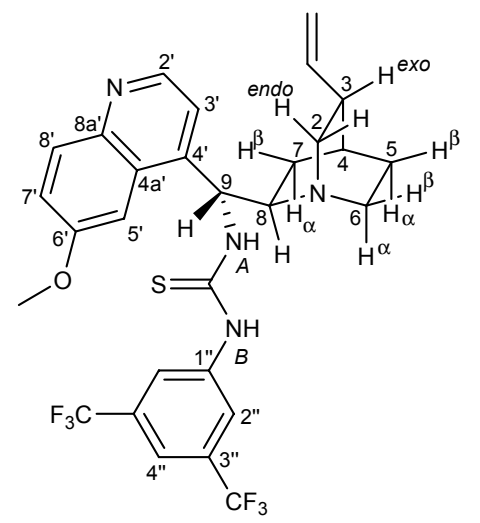

7. Amorphous solid. Yield: $69 \%$. $[\alpha]_{\mathrm{D}}^{25}+225.3\left(c 0.50, \mathrm{CHCl}_{3}\right)$; ${ }^{1} \mathrm{H}-\mathrm{NMR}\left(500 \mathrm{MHz}, \mathrm{CD}_{3} \mathrm{OD}, \mathrm{T}=310 \mathrm{~K}, \delta_{\mathrm{TMS}}=0 \mathrm{ppm}\right) \delta 8.67(\mathrm{~d}, J$ $=4.7 \mathrm{~Hz}, 1 \mathrm{H}, \mathrm{H}-2$ '), 8.11 (br s, 2H, 2H-2'’), 8.03 (br d, $J=2.6 \mathrm{~Hz}$, 1H, H-5'), 7.94 (d, $J=9.3$ Hz, 1H, H-8'), 7.59 (br s, 1H, H-4'’), 7.56 (d, $J=4.7 \mathrm{~Hz}, 1 \mathrm{H}, \mathrm{H}-3$ '), 7.43 (dd, $J=9.3,2.6 \mathrm{~Hz}, 1 \mathrm{H}, \mathrm{H}-7^{\prime}$ ), 6.35 
(d, $J=11.0 \mathrm{~Hz}, 1 \mathrm{H}, \mathrm{H}-9), 5.96$ (ddd, $J=17.2,10.5,6.2 \mathrm{~Hz}, 1 \mathrm{H}, \mathrm{C} H=\mathrm{CH}_{2}$ ), 5.22 (dt, $J=10.5,1.5$ $\left.\mathrm{Hz}, 1 \mathrm{H}, \mathrm{CH}=\mathrm{CH}_{2}\right), 5.15\left(\mathrm{dt}, J=17.2,1.5 \mathrm{~Hz}, 1 \mathrm{H}, \mathrm{CH}=\mathrm{CH}_{2}\right), 4.03\left(\mathrm{~s}, 3 \mathrm{H}, \mathrm{OCH}_{3}\right), 3.04(\mathrm{dd}, J=$ 13.6, $9.9 \mathrm{~Hz}, 1 \mathrm{H}, \mathrm{H}-2$-exo), 3.34 (ddd, $J=13.6,4.7,2.3 \mathrm{~Hz}, 1 \mathrm{H}, \mathrm{H}-2$-endo), 3.33 (pseudo q, $J=$ $10.7 \mathrm{~Hz}, 1 \mathrm{H}, \mathrm{H}-8$ ), 3.01 (m, 2H, H-6 $\alpha$ and H-63), 2.37 (br m, 1H, H-3), 1.63 (br m, 1H, H-4), 1.60

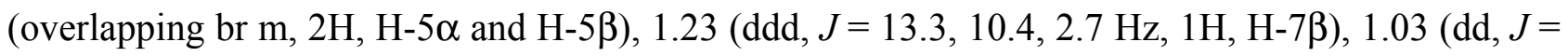
13.3, $10.4 \mathrm{~Hz}, 1 \mathrm{H}, \mathrm{H}-7 \alpha) \mathrm{ppm} ;{ }^{13} \mathrm{C}-\mathrm{NMR}\left(125 \mathrm{MHz}, \mathrm{CD}_{3} \mathrm{OD}, \mathrm{T}=310 \mathrm{~K}, \delta_{\mathrm{TMS}}=0 \mathrm{ppm}\right) \delta 181.7$ (C=S), 158.6 (C-6'), 147.3 (C-2'), 146.9 (C-4'), 144.2 (C-8a'), 142.1 (C-1'’), $140.8\left(\mathrm{CH}^{\prime}=\mathrm{CH}_{2}\right)$, $131.8\left(\mathrm{q},{ }^{2} J_{\mathrm{CF}}=33.0 \mathrm{~Hz}, \mathrm{C}-3\right.$ ') $), 130.2\left(\mathrm{C}-8\right.$ ') $129.2(\mathrm{C}-4 \mathrm{a}), 123.7$ (q, $\left.{ }^{1} J_{\mathrm{CF}}=272.2 \mathrm{~Hz}, \mathrm{CF}_{3}\right), 122.9$ (C-2'), 122.7 (coalesced lines, C-3' and C-7'), 116.9 (br signal, ${ }^{3} J_{\mathrm{CF}}=3.7 \mathrm{~Hz}, \mathrm{C}-4$ '), 114.3 $\left(\mathrm{CH}=\mathrm{CH}_{2}\right), 103.8\left(\mathrm{C}-5\right.$ '), $60.7(\mathrm{C}-8), 55.5\left(\mathrm{OCH}_{3}\right), 54.6(\mathrm{C}-9), 49.2(\mathrm{C}-6), 47.6(\mathrm{C}-2), 39.2(\mathrm{C}-3)$, 27.7 (C-4), 26.4 (C-5), 25.5 (C-7) ppm; ${ }^{15} \mathrm{~N}-\mathrm{NMR}\left(50 \mathrm{MHz}, \mathrm{CD}_{3} \mathrm{OD}, \mathrm{T}=310 \mathrm{~K}, \delta_{\mathrm{NH}_{3}}=0 \mathrm{ppm}\right) \delta$ 25 (quinuclidine-N), 294 (quinoline-N), $131\left(\mathrm{~N}_{\mathrm{A}}\right), 124\left(\mathrm{~N}_{\mathrm{B}}\right)$ ppm; IR $(\mathrm{KBr}) \vee$ 1622, 1511, 1475, $1384,1278,1172,1131,1033,886,682 \mathrm{~cm}^{-1}$; HRMS (EI) Exact mass calculated for $\mathrm{C}_{29} \mathrm{H}_{28} \mathrm{~F}_{6} \mathrm{~N}_{4} \mathrm{OS}$ $[\mathrm{M}]^{+}$594.1888; Found: 594.1878 .

\section{Representative procedure for enantioselective Michael addition of nitromethane to chalcones. Reaction of p-fluorochalcone $2 \mathrm{c}$ is typical.}

Toluene (3.0 mL), p-fluorochalcone $2 \mathrm{c}(1.13 \mathrm{~g}, 5.0 \mathrm{mmol})$ and nitromethane (1) $(5.0 \mathrm{eq}, 1.53 \mathrm{~g})$ were loaded in capped vial. Then thiourea catalyst $5 \mathbf{b}(298 \mathrm{mg}, 0.50 \mathrm{mmol})$ was added to the mixture and stirring was maintained for $122 \mathrm{~h}$ at room temperature. The volatiles were removed in vacuo and the residue was purified by column chromatography on silica gel (hexane/EtOAc $=3 / 1$ as eluant) affording Michael adduct $\mathbf{3 c}$ as a white solid.

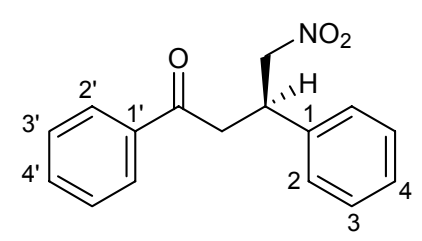

3a. ${ }^{5}$ (Only 3.0 eq of nitromethane was added.) $[\alpha]_{\mathrm{D}}{ }^{25}+26.7$ (c 1.00 , $\mathrm{CHCl}_{3}, 96 \%$ ee); ${ }^{1} \mathrm{H}-\mathrm{NMR}\left(500 \mathrm{MHz}, \mathrm{CDCl}_{3}, \mathrm{~T}=300 \mathrm{~K}, \delta_{\mathrm{TMS}}=0 \mathrm{ppm}\right)$ $\delta 7.93$ (d, $J=7.9 \mathrm{~Hz}, 2 \mathrm{H}, \mathrm{H}-2$ ' and H-6'), 7.59 (t, $J=7.2 \mathrm{~Hz}, 1 \mathrm{H}, \mathrm{H}-4$ '),

7.47 (t, $J=7.2 \mathrm{~Hz}, 2 \mathrm{H}, \mathrm{H}-3$ ' and H-5'), 7.35 (t, $J=7.2 \mathrm{~Hz}, 2 \mathrm{H}, \mathrm{H}-3$ and H-5), 7.31 (d, $J=7.2 \mathrm{~Hz}, 2 \mathrm{H}, \mathrm{H}-2$ and H-6), 7.28 (t, $J=7.2 \mathrm{~Hz}, 1 \mathrm{H}, \mathrm{H}-4), 4.85$ (ABX, $J_{\mathrm{AB}}=12.6$ $\left.\mathrm{Hz}, J_{\mathrm{AX}}=6.6 \mathrm{~Hz}, J_{\mathrm{BX}}=8.1 \mathrm{~Hz}, 1 \mathrm{H}, \mathrm{CH}_{2} \mathrm{NO}_{2}\right), 4.71\left(\mathrm{ABX}, J_{\mathrm{AB}}=12.6 \mathrm{~Hz}, J_{\mathrm{AX}}=6.6 \mathrm{~Hz}, J_{\mathrm{BX}}=8.1\right.$ 
$\mathrm{Hz}, 1 \mathrm{H}, \mathrm{CH}_{2} \mathrm{NO}_{2}$ ), 4.25 (br pseudo quintet, $\left.J=7.1 \mathrm{~Hz}, 1 \mathrm{H}, \mathrm{CH}\right), 3.49\left(\mathrm{ABX}, J_{\mathrm{AB}}=17.4 \mathrm{~Hz}, J_{\mathrm{AX}}=\right.$ $\left.6.5 \mathrm{~Hz}, J_{\mathrm{BX}}=8.0 \mathrm{~Hz}, 1 \mathrm{H}, \mathrm{CH}_{2} \mathrm{CO}\right), 3.45\left(\mathrm{ABX}, J_{\mathrm{AB}}=17.4 \mathrm{~Hz}, J_{\mathrm{AX}}=6.5 \mathrm{~Hz}, J_{\mathrm{BX}}=8.0 \mathrm{~Hz}, 1 \mathrm{H}\right.$, $\left.\mathrm{CH}_{2} \mathrm{CO}\right) \mathrm{ppm} ;{ }^{13} \mathrm{C}-\mathrm{NMR}\left(125 \mathrm{MHz}, \mathrm{CDCl}_{3}, \mathrm{~T}=300 \mathrm{~K}, \delta_{\mathrm{TMS}}=0 \mathrm{ppm}\right) \delta 196.8(\mathrm{C}=\mathrm{O}), 139.1(\mathrm{C}-1)$, 136.4 (C-1'), 133.5 (C-4'), 129.0 (C-3,5), 128.7 (C-3',5’), 128.0 (C-2',6’), 127.8 (C-4), 127.4 (C2,6), $79.5\left(\mathrm{CH}_{2} \mathrm{NO}_{2}\right), 41.5\left(\mathrm{CH}_{2} \mathrm{CO}\right), 39.3(\mathrm{CH}) \mathrm{ppm} ;{ }^{15} \mathrm{~N}-\mathrm{NMR}\left(50 \mathrm{MHz}, \mathrm{CDCl}_{3}, \mathrm{~T}=300 \mathrm{~K}\right.$, $\left.\delta_{\mathrm{NH}_{3}}=0 \mathrm{ppm}\right) \delta 383\left(\mathrm{NO}_{2}\right) \mathrm{ppm} ; 96 \%$ ee Enantioselectivity was determined by HPLC analysis with a Chiralpak AD column, 10\% isopropyl alcohol in hexane, $1.0 \mathrm{~mL} / \mathrm{min}, \lambda=254 \mathrm{~nm}$, retention times minor $14.9 \mathrm{~min}$, major $19.8 \mathrm{~min}$.

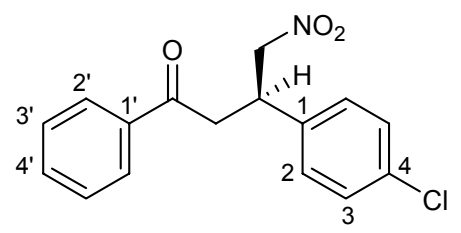

3b. ${ }^{6} \quad$ The crude product was purified by flash chromatography on silica gel (hexane/ EtOAc $=3 / 1$ as eluant). ${ }^{1} \mathrm{H}-\mathrm{NMR}(500 \mathrm{MHz}$, $\left.\mathrm{CDCl}_{3}, \mathrm{~T}=300 \mathrm{~K}, \delta_{\mathrm{TMS}}=0 \mathrm{ppm}\right) \delta 7.92(\mathrm{~d}, J=7.9 \mathrm{~Hz}, 2 \mathrm{H}, \mathrm{H}-2$ ' and H-6'), 7.59 (t, $J=7.2 \mathrm{~Hz}, 1 \mathrm{H}, \mathrm{H}-4$ '), 7.47 (t, $J=7.2 \mathrm{~Hz}, 2 \mathrm{H}, \mathrm{H}-3$ ' and H-5'), 7.31 (t, $J=8.0 \mathrm{~Hz}, 2 \mathrm{H}, \mathrm{H}-3$ and H-5), 7.24 (d, $J=8.0 \mathrm{~Hz}, 2 \mathrm{H}, \mathrm{H}-2$ and H-6), 4.82 (ABX, $J_{\mathrm{AB}}$ $\left.=12.6 \mathrm{~Hz}, J_{\mathrm{AX}}=6.6 \mathrm{~Hz}, J_{\mathrm{BX}}=8.1 \mathrm{~Hz}, 1 \mathrm{H}, \mathrm{CH}_{2} \mathrm{NO}_{2}\right), 4.67\left(\mathrm{ABX}, J_{\mathrm{AB}}=12.6 \mathrm{~Hz}, J_{\mathrm{AX}}=6.6 \mathrm{~Hz}, J_{\mathrm{BX}}\right.$ $\left.=8.1 \mathrm{~Hz}, 1 \mathrm{H}, \mathrm{CH}_{2} \mathrm{NO}_{2}\right), 4.23$ (br pseudo quintet, $\left.J=7.1 \mathrm{~Hz}, 1 \mathrm{H}, \mathrm{CH}\right), 3.46\left(\mathrm{ABX}, J_{\mathrm{AB}}=17.4 \mathrm{~Hz}\right.$, $\left.J_{\mathrm{AX}}=6.5 \mathrm{~Hz}, J_{\mathrm{BX}}=8.0 \mathrm{~Hz}, 1 \mathrm{H}, \mathrm{CH}_{2} \mathrm{CO}\right), 3.43\left(\mathrm{ABX}, J_{\mathrm{AB}}=17.4 \mathrm{~Hz}, J_{\mathrm{AX}}=6.5 \mathrm{~Hz}, J_{\mathrm{BX}}=8.0 \mathrm{~Hz}\right.$, $\left.1 \mathrm{H}, \mathrm{CH}_{2} \mathrm{CO}\right) \mathrm{ppm} ;{ }^{13} \mathrm{C}-\mathrm{NMR}\left(125 \mathrm{MHz}, \mathrm{CDCl}_{3}, \mathrm{~T}=300 \mathrm{~K}, \delta_{\mathrm{TMS}}=0 \mathrm{ppm}\right) \delta 196.5(\mathrm{C}=\mathrm{O}), 137.6$ (C-1), 136.2 (C-1'), 133.7 (C-4'), 132.2 (C-4), 129.2 (C-3,5), 128.9 (C-2,6), 128.7 (C-3',5'), 128.0 (C-2',6'), $79.3\left(\mathrm{CH}_{2} \mathrm{NO}_{2}\right), 41.3\left(\mathrm{CH}_{2} \mathrm{CO}\right), 38.7(\mathrm{CH}) \mathrm{ppm} ;{ }^{15} \mathrm{~N}-\mathrm{NMR}\left(50 \mathrm{MHz}, \mathrm{CDCl}_{3}, \mathrm{~T}=300 \mathrm{~K}\right.$, $\left.\delta_{\mathrm{NH}_{3}}=0 \mathrm{ppm}\right) \delta 383\left(\mathrm{NO}_{2}\right)$ ppm; 95\% ee Enantioselectivity was determined by HPLC analysis with a Chiralpak AD column, 10\% isopropyl alcohol in hexane, $1.0 \mathrm{~mL} / \mathrm{min}, \lambda=254 \mathrm{~nm}$, retention times minor $17.9 \mathrm{~min}$, major $25.5 \mathrm{~min}$.

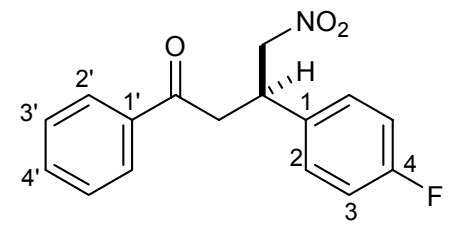

3c. Slightly yellow oil. $[\alpha]_{\mathrm{D}}^{25}+26.7$ (c $1.00, \mathrm{CHCl}_{3}, 98 \%$ ee); mp $42-43{ }^{\circ} \mathrm{C} ;{ }^{1} \mathrm{H}-\mathrm{NMR}\left(500 \mathrm{MHz}, \mathrm{CDCl}_{3}, \mathrm{~T}=300 \mathrm{~K}, \delta_{\mathrm{TMS}}=0 \mathrm{ppm}\right) \delta$ 7.93 (d, $J=7.9 \mathrm{~Hz}, 2 \mathrm{H}, \mathrm{H}-2$ ' and H-6'), 7.59 (t, $J=7.2 \mathrm{~Hz}, 1 \mathrm{H}, \mathrm{H}-$ 4'), 7.47 (t, $J=7.2 \mathrm{~Hz}, 2 \mathrm{H}, \mathrm{H}-3$ ' and H-5'), 7.28 (dd, $J=8.7,5.3 \mathrm{~Hz}$, $2 \mathrm{H}, \mathrm{H}-2$ and $\mathrm{H}-6), 7.03(\mathrm{t}, J=8.7 \mathrm{~Hz}, 2 \mathrm{H}, \mathrm{H}-3$ and $\mathrm{H}-5), 4.83\left(\mathrm{ABX}, J_{\mathrm{AB}}=12.6 \mathrm{~Hz}, J_{\mathrm{AX}}=6.6 \mathrm{~Hz}\right.$, 
$\left.J_{\mathrm{BX}}=8.1 \mathrm{~Hz}, 1 \mathrm{H}, \mathrm{CH}_{2} \mathrm{NO}_{2}\right), 4.67\left(\mathrm{ABX}, J_{\mathrm{AB}}=12.6 \mathrm{~Hz}, J_{\mathrm{AX}}=6.6 \mathrm{~Hz}, J_{\mathrm{BX}}=8.1 \mathrm{~Hz}, 1 \mathrm{H}, \mathrm{CH}_{2} \mathrm{NO}_{2}\right)$, 4.24 (br pseudo quintet, $J=7.1 \mathrm{~Hz}, 1 \mathrm{H}, \mathrm{CH}), 3.46\left(\mathrm{ABX}, J_{\mathrm{AB}}=17.4 \mathrm{~Hz}, J_{\mathrm{AX}}=6.5 \mathrm{~Hz}, J_{\mathrm{BX}}=8.0\right.$ $\left.\mathrm{Hz}, 1 \mathrm{H}, \mathrm{CH}_{2} \mathrm{CO}\right), 3.43\left(\mathrm{ABX}, J_{\mathrm{AB}}=17.4 \mathrm{~Hz}, J_{\mathrm{AX}}=6.5 \mathrm{~Hz}, J_{\mathrm{BX}}=8.0 \mathrm{~Hz}, 1 \mathrm{H}, \mathrm{CH}_{2} \mathrm{CO}\right) \mathrm{ppm} ;{ }^{13} \mathrm{C}-$ NMR $\left(125 \mathrm{MHz}, \mathrm{CDCl}_{3}, \mathrm{~T}=300 \mathrm{~K}, \delta_{\mathrm{TMS}}=0 \mathrm{ppm}\right) \delta 196.6(\mathrm{C}=\mathrm{O}), 162.2\left(\mathrm{~d},{ }^{1} J_{\mathrm{CF}}=246.5 \mathrm{~Hz}, \mathrm{C}-4\right)$, $136.3\left(\mathrm{C}-1^{\prime}\right), 134.9\left(\mathrm{~d},{ }^{4} J_{\mathrm{CF}}=3.7 \mathrm{~Hz}, \mathrm{C}-1\right), 133.6\left(\mathrm{C}-4^{\prime}\right), 129.1$ (d, $\left.{ }^{3} J_{\mathrm{CF}}=8.2 \mathrm{~Hz}, \mathrm{C}-2,6\right), 128.8(\mathrm{C}-$ 3',5'), $128.0\left(\mathrm{C}-2^{\prime}, 6^{\prime}\right), 116.0\left(\mathrm{~d},{ }^{2} J_{\mathrm{CF}}=21.1 \mathrm{~Hz}, \mathrm{C}-3,5\right), 79.6\left(\mathrm{CH}_{2} \mathrm{NO}_{2}\right), 41.5\left(\mathrm{CH}_{2} \mathrm{CO}\right), 38.6(\mathrm{CH})$ ppm; ${ }^{15} \mathrm{~N}-\mathrm{NMR}\left(50 \mathrm{MHz}, \mathrm{CDCl}_{3}, \mathrm{~T}=300 \mathrm{~K}, \delta_{\mathrm{NH}_{3}}=0 \mathrm{ppm}\right) \delta 382\left(\mathrm{NO}_{2}\right) \mathrm{ppm}$; IR $(\mathrm{KBr}) \mathrm{v} 1685$, 1545, 1512, 1364, 1230, 1162, 840, 685, $557 \mathrm{~cm}^{-1}$; Anal. Calcd. for $\mathrm{C}_{16} \mathrm{H}_{14} \mathrm{FNO}_{3}$ : C, 66.89; H, 4.91; F, 6.61; N, 4.88; O, 16.71. Found: C, 67.01; H, 5.18; N, 4.79. Enantioselectivity was determined by HPLC analysis with a Chiralpak AD column, 10\% isopropyl alcohol in hexane, $1.0 \mathrm{~mL} / \mathrm{min}, \lambda=$ $254 \mathrm{~nm}$, retention times minor $16.8 \mathrm{~min}$, major $23.1 \mathrm{~min}$.

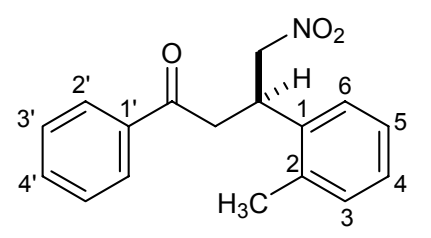

3d. Slightly yellow oil. $[\alpha]_{\mathrm{D}}{ }^{25}+40.1$ (c $1.00, \mathrm{CHCl}_{3}, 89 \%$ ee); ${ }^{1} \mathrm{H}-$ NMR $\left(500 \mathrm{MHz}, \mathrm{CDCl}_{3}, \mathrm{~T}=300 \mathrm{~K}, \delta_{\mathrm{TMS}}=0 \mathrm{ppm}\right) \delta 7.93(\mathrm{~d}, J=7.9$ Hz, 2H, H-2' and H-6'), 7.59 (t, $J=7.2 \mathrm{~Hz}, 1 \mathrm{H}, \mathrm{H}-4$ '), 7.47 (t, $J=7.2$ $\mathrm{Hz}, 2 \mathrm{H}, \mathrm{H}-3^{\prime}$ and $\left.\mathrm{H}-5^{\prime}\right), 7.14-7.23$ (m, 4H,H-2-5), $4.80\left(\mathrm{ABX}, J_{\mathrm{AB}}=\right.$ $\left.12.6 \mathrm{~Hz}, J_{\mathrm{AX}}=6.6 \mathrm{~Hz}, J_{\mathrm{BX}}=8.1 \mathrm{~Hz}, 1 \mathrm{H}, \mathrm{CH}_{2} \mathrm{NO}_{2}\right), 4.68\left(\mathrm{ABX}, J_{\mathrm{AB}}=12.6 \mathrm{~Hz}, J_{\mathrm{AX}}=6.6 \mathrm{~Hz}, J_{\mathrm{BX}}=\right.$ $\left.8.1 \mathrm{~Hz}, \mathrm{CH}_{2} \mathrm{NO}_{2}\right), 4.55$ (br pseudo quintet, $\left.J=7.1 \mathrm{~Hz}, 1 \mathrm{H}, \mathrm{CH}\right), 3.49\left(\mathrm{ABX}, J_{\mathrm{AB}}=17.4 \mathrm{~Hz}, J_{\mathrm{AX}}=\right.$ $\left.6.5 \mathrm{~Hz}, J_{\mathrm{BX}}=8.0 \mathrm{~Hz}, 1 \mathrm{H}, \mathrm{CH}_{2} \mathrm{CO}\right), 3.41\left(\mathrm{ABX}, J_{\mathrm{AB}}=17.4 \mathrm{~Hz}, J_{\mathrm{AX}}=6.5 \mathrm{~Hz}, J_{\mathrm{BX}}=8.0 \mathrm{~Hz}, 1 \mathrm{H}\right.$, $\left.\mathrm{CH}_{2} \mathrm{CO}\right), 2.49\left(\mathrm{~s}, 3 \mathrm{H}, \mathrm{CH}_{3}\right) \mathrm{ppm} ;{ }^{13} \mathrm{C}-\mathrm{NMR}\left(125 \mathrm{MHz}, \mathrm{CDCl}_{3}, \mathrm{~T}=300 \mathrm{~K}, \delta_{\mathrm{TMS}}=0 \mathrm{ppm}\right) \delta 196.9$ (C=O), 137.4 (C-1), 136.5 (C-2,6), 133.5 (C-4'), 131.2 (C-3,5), 128.7 (C-3’,5'), 128.0 (C-2',6'), 127.5 (C-3,5), 126.6 (C-4), $125.4(\mathrm{C}-2,6), 79.0\left(\mathrm{CH}_{2} \mathrm{NO}_{2}\right), 41.6\left(\mathrm{CH}_{2} \mathrm{CO}\right), 34.4(\mathrm{CH}), 19.6\left(\mathrm{CH}_{3}\right)$ ppm; ${ }^{15} \mathrm{~N}-\mathrm{NMR}\left(50 \mathrm{MHz}, \mathrm{CDCl}_{3}, \mathrm{~T}=300 \mathrm{~K}, \delta_{\mathrm{NH}_{3}}=0 \mathrm{ppm}\right) \delta 384\left(\mathrm{NO}_{2}\right) \mathrm{ppm} ; \mathrm{IR}\left(\mathrm{CH}_{2} \mathrm{Cl}_{2}\right) \vee 1688$, 1555, 1449, 1422, 1378, 1265, $896 \mathrm{~cm}^{-1}$; HRMS (EI) Exact mass calculated for $\mathrm{C}_{17} \mathrm{H}_{17} \mathrm{NO}_{3}[\mathrm{M}]^{+}$ 283.1208; Found: 287.1208; Anal. Calcd. for $\mathrm{C}_{17} \mathrm{H}_{17} \mathrm{NO}_{3}$ : C, 72.07; H, 6.05; F, 6.61; N, 4.94; O, 16.94. Found: C, 71.75; H, 6.03; N, 4.87. Enantioselectivity was determined by HPLC analysis with a Chiralpak AD column, 10\% isopropyl alcohol in hexane, $1.0 \mathrm{~mL} / \mathrm{min}, \lambda=254 \mathrm{~nm}$, retention times minor $10.7 \mathrm{~min}$, major $13.3 \mathrm{~min}$. 


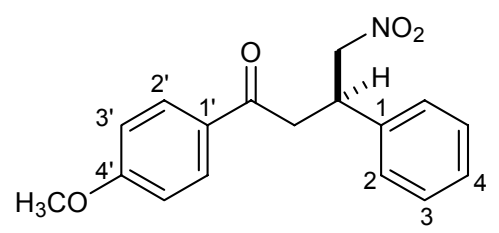

3e. ${ }^{7}[\alpha]_{\mathrm{D}}^{25}+40.0\left(c 1.00, \mathrm{CHCl}_{3}, 96 \%\right.$ ee $){ }^{1} \mathrm{H}-\mathrm{NMR}(500 \mathrm{MHz}$, $\left.\mathrm{CDCl}_{3}, \mathrm{~T}=300 \mathrm{~K}, \delta_{\mathrm{TMS}}=0 \mathrm{ppm}\right) \delta 7.91(\mathrm{~d}, J=9.0 \mathrm{~Hz}, 2 \mathrm{H}, \mathrm{H}-2$ ' and H-6'), 7.35 (t, $J=7.2 \mathrm{~Hz}, 2 \mathrm{H}, \mathrm{H}-3$ and $\mathrm{H}-5), 7.31$ (d, $J=7.2$ $\mathrm{Hz}, 2 \mathrm{H}, \mathrm{H}-2$ and $\mathrm{H}-6), 7.28$ (t, $J=7.2 \mathrm{~Hz}, 1 \mathrm{H}, \mathrm{H}-4), 6.91$ (d, $J=9.0$

$\mathrm{Hz}, 2 \mathrm{H}, \mathrm{H}-3^{\prime}$ and $\mathrm{H}-5$ ') $, 4.85\left(\mathrm{ABX}, J_{\mathrm{AB}}=12.6 \mathrm{~Hz}, J_{\mathrm{AX}}=6.6 \mathrm{~Hz}, J_{\mathrm{BX}}=8.1 \mathrm{~Hz}, 1 \mathrm{H}, \mathrm{CH}_{2} \mathrm{NO}_{2}\right), 4.69$ $\left(\mathrm{ABX}, J_{\mathrm{AB}}=12.6 \mathrm{~Hz}, J_{\mathrm{AX}}=6.6 \mathrm{~Hz}, J_{\mathrm{BX}}=8.1 \mathrm{~Hz}, 1 \mathrm{H}, \mathrm{CH}_{2} \mathrm{NO}_{2}\right.$ ), 4.22 (br pseudo quintet, $J=7.1$ $\mathrm{Hz}, 1 \mathrm{H}, \mathrm{CH}), 3.87\left(\mathrm{~s}, 3 \mathrm{H}, \mathrm{OCH}_{3}\right), 3.42\left(\mathrm{ABX}, J_{\mathrm{AB}}=17.4 \mathrm{~Hz}, J_{\mathrm{AX}}=6.5 \mathrm{~Hz}, J_{\mathrm{BX}}=8.0 \mathrm{~Hz}, 1 \mathrm{H}\right.$, $\left.\mathrm{CH}_{2} \mathrm{CO}\right), 3.38\left(\mathrm{ABX}, J_{\mathrm{AB}}=17.4 \mathrm{~Hz}, J_{\mathrm{AX}}=6.5 \mathrm{~Hz}, J_{\mathrm{BX}}=8.0 \mathrm{~Hz}, 1 \mathrm{H}, \mathrm{CH}_{2} \mathrm{CO}\right) \mathrm{ppm} ;{ }^{13} \mathrm{C}-\mathrm{NMR}(125$ $\left.\mathrm{MHz}, \mathrm{CDCl}_{3}, \mathrm{~T}=300 \mathrm{~K}, \delta_{\mathrm{TMS}}=0 \mathrm{ppm}\right) \delta 195.3(\mathrm{C}=\mathrm{O}), 163.8\left(\mathrm{C}-4\right.$ '), $139.3(\mathrm{C}-1), 130.3\left(\mathrm{C}-2^{\prime}, 6^{\prime}\right)$, 129.5 (C-1'), 129.0 (C-3,5), 127.7 (C-4), 127.4 (C-2,6), 113.8 (C-3',5'), $79.6\left(\mathrm{CH}_{2} \mathrm{NO}_{2}\right), 55.5$ $\left(\mathrm{OCH}_{3}\right), 41.1\left(\mathrm{CH}_{2} \mathrm{CO}\right), 39.4(\mathrm{CH}) \mathrm{ppm} ;{ }^{15} \mathrm{~N}-\mathrm{NMR}\left(50 \mathrm{MHz}, \mathrm{CDCl}_{3}, \mathrm{~T}=300 \mathrm{~K}, \delta_{\mathrm{NH}_{3}}=0 \mathrm{ppm}\right) \delta$ $382\left(\mathrm{NO}_{2}\right)$ ppm; Enantioselectivity was determined by HPLC analysis with a Chiralpak AD column, $10 \%$ isopropyl alcohol in hexane, $1.0 \mathrm{~mL} / \mathrm{min}, \lambda=254 \mathrm{~nm}$, retention times minor $30.8 \mathrm{~min}$, major $47.2 \mathrm{~min}$.

\section{References:}

${ }^{1}$ (a) Dannhardt, G.; Kiefer, W.; Kraemer, G.; Maehrlein, S.; Nowe, U.; Fiebich, B. Eur. J. Med. Chem. Chim. Ther. 1991, 34, 2804. (b) Frank, R. L.; Seven, R. P. J. Am. Chem. Soc. 1949, 71, 2629. (c) Hine, J.; Skoglund, M. J. J. Org. Chem. 1982, 47, 4758. (d) Kellogg, R. M.; Nieuwenhuijzen, J. W.; Pouwer, K.; Vries, T. R.; Broxterman, Q. B.; Grimbergen, R. F. P.; Kaptein, B.; Crois, R. M. La; Wever, E. de; Zwaagastra, K.; Laan, A. C. van der Synthesis 2003, 1626.

${ }^{2}$ Brunner, H.; Bügler, J.; Nuber, B. Tetrahedron: Asymmetry 1995, 6, 1699.

${ }^{3}$ Brunner, H.; Schmidt, P. Eur. J. Org. Chem. 2000, 2119.

${ }^{4}$ Brunner, H.; Bügler, J. Bull. Soc. Chim. Belg. 1997, 106, 77.

${ }^{5}$ Botteghi, C.; Paganelli, S.; Schionato, A.; Boga, C.; Fava, A. J. Mol. Catal. 1991, 66, 7.

${ }^{6}$ Corey, E. J.; Zhang, F.-Y., Org. Lett. $2000,2,4257$.

${ }^{7}$ Worrall, D. E.; Bradway, C. J., J. Am. Chem. Soc. 1936, 58, 1607. 


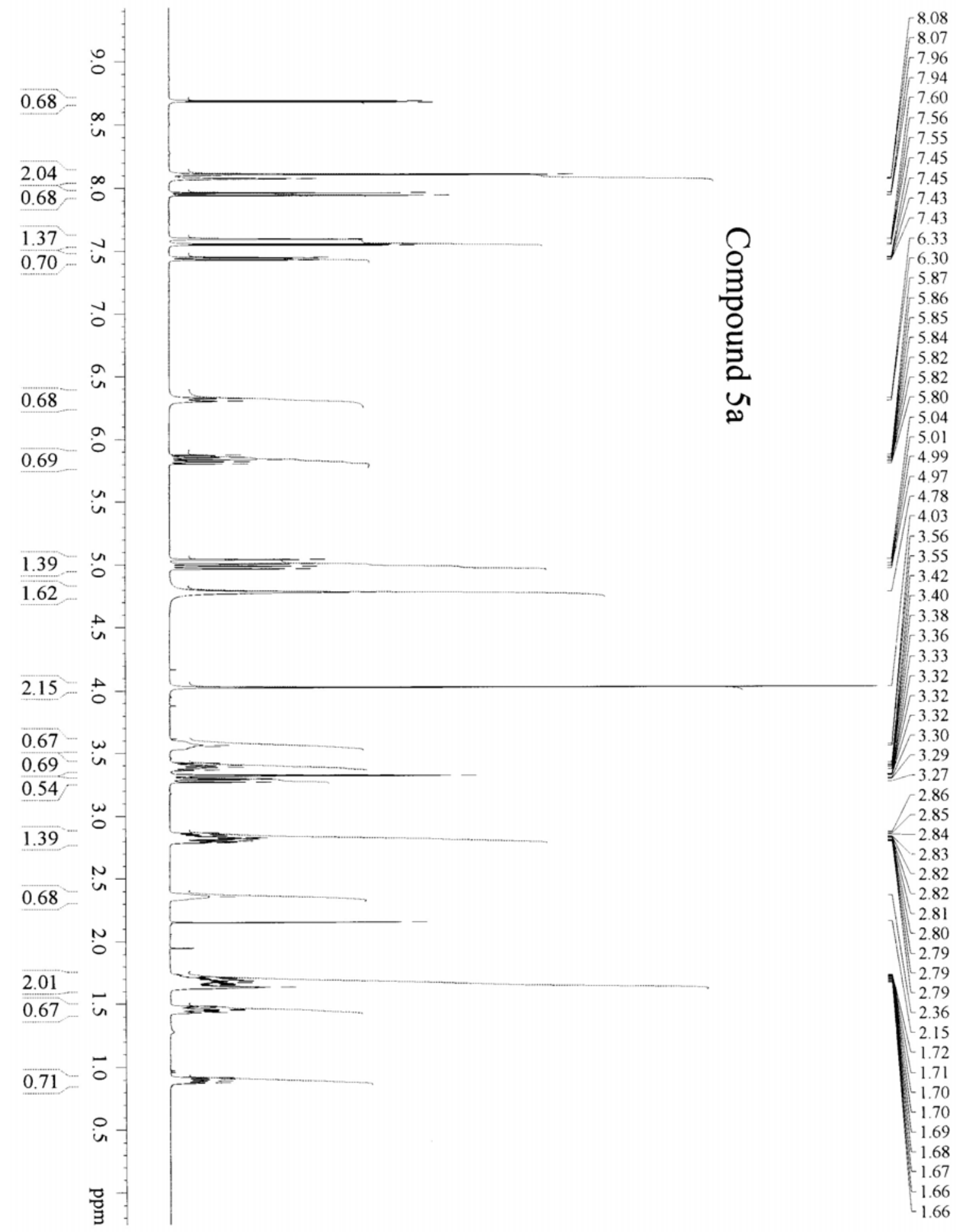

Figure 1. ${ }^{1} \mathrm{H}$ NMR spectra of 5 a 


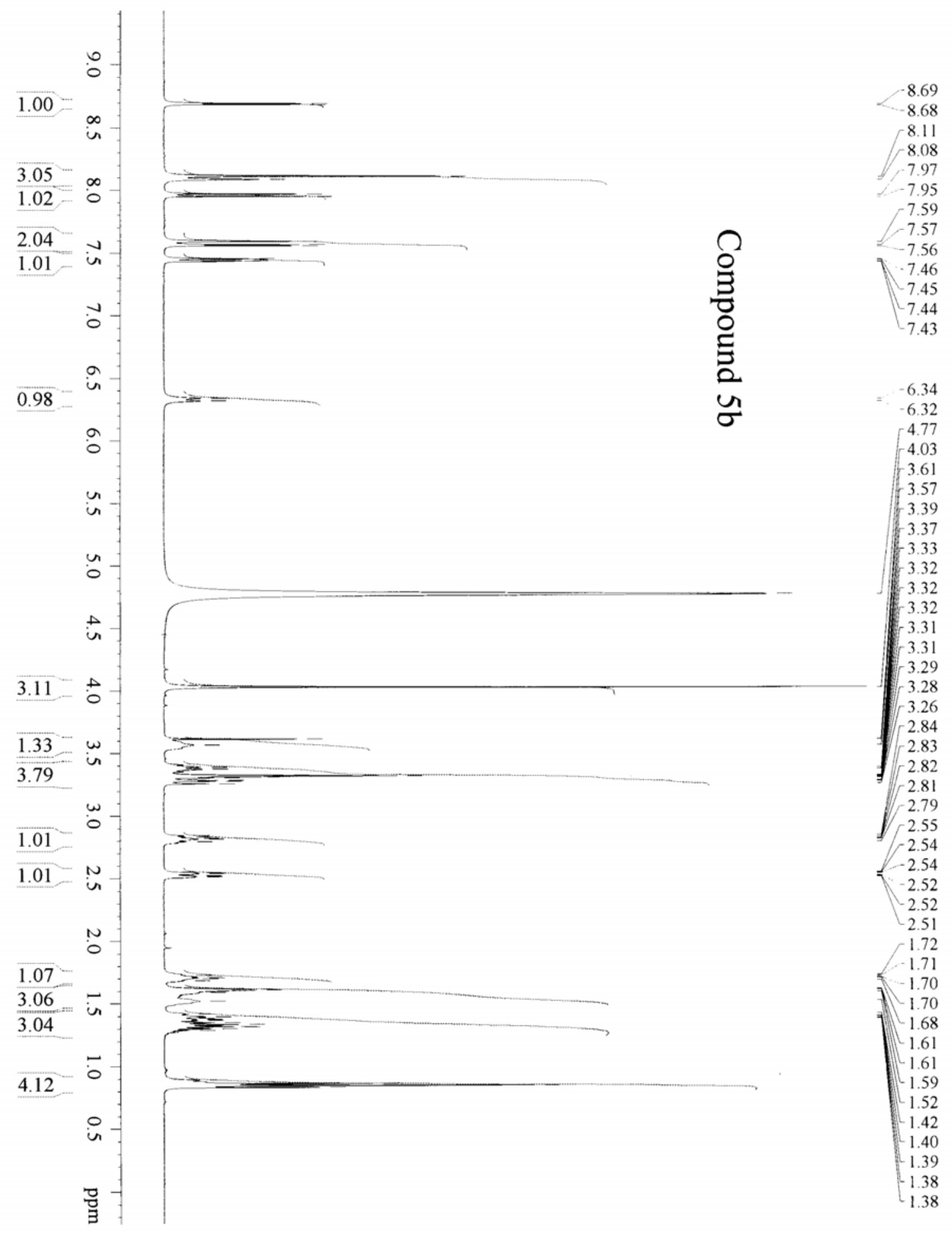

Figure 2. ${ }^{1} \mathrm{H}$ NMR spectra of $5 b$ 


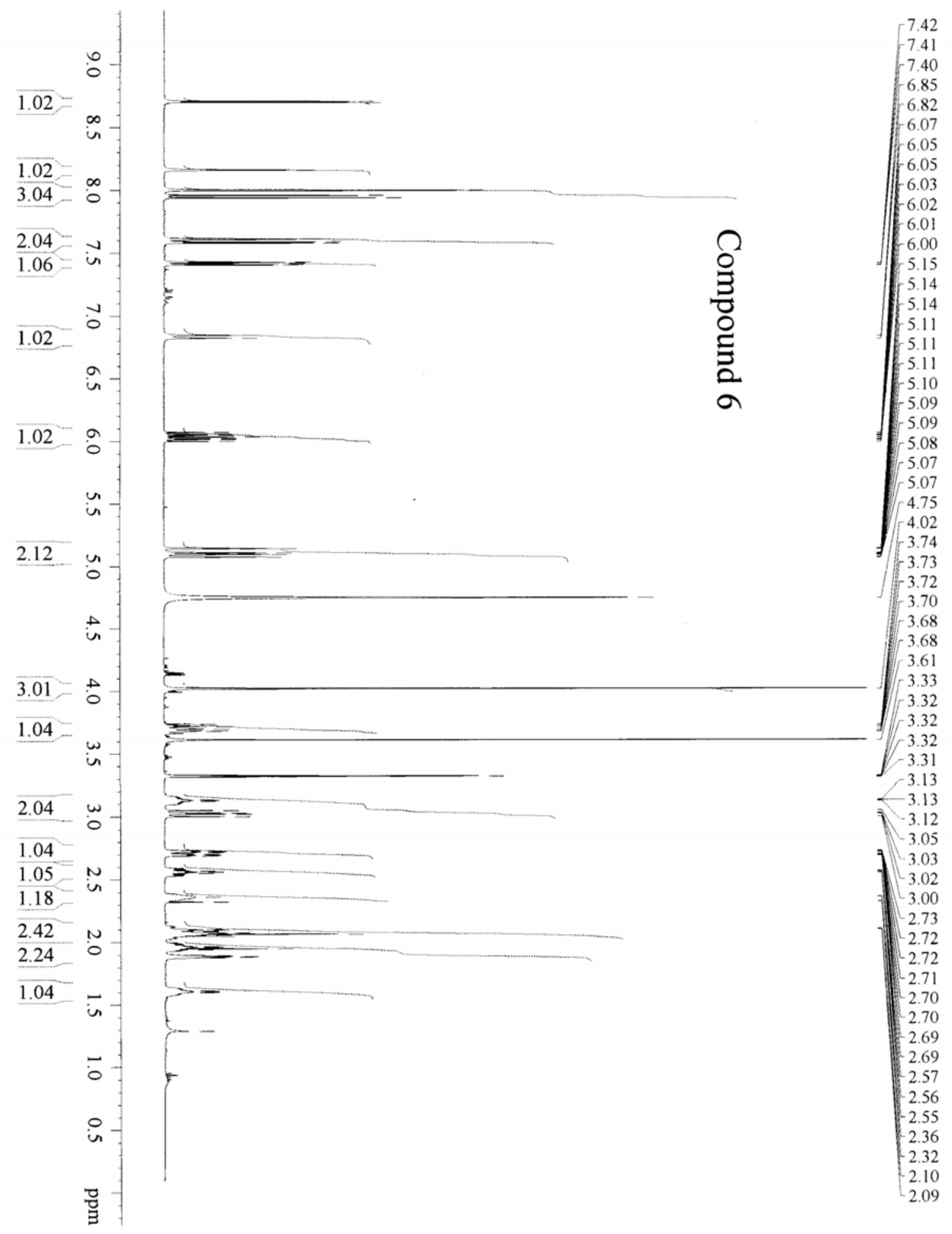

Figure 3. ${ }^{1} \mathrm{H}$ NMR spectra of 6 


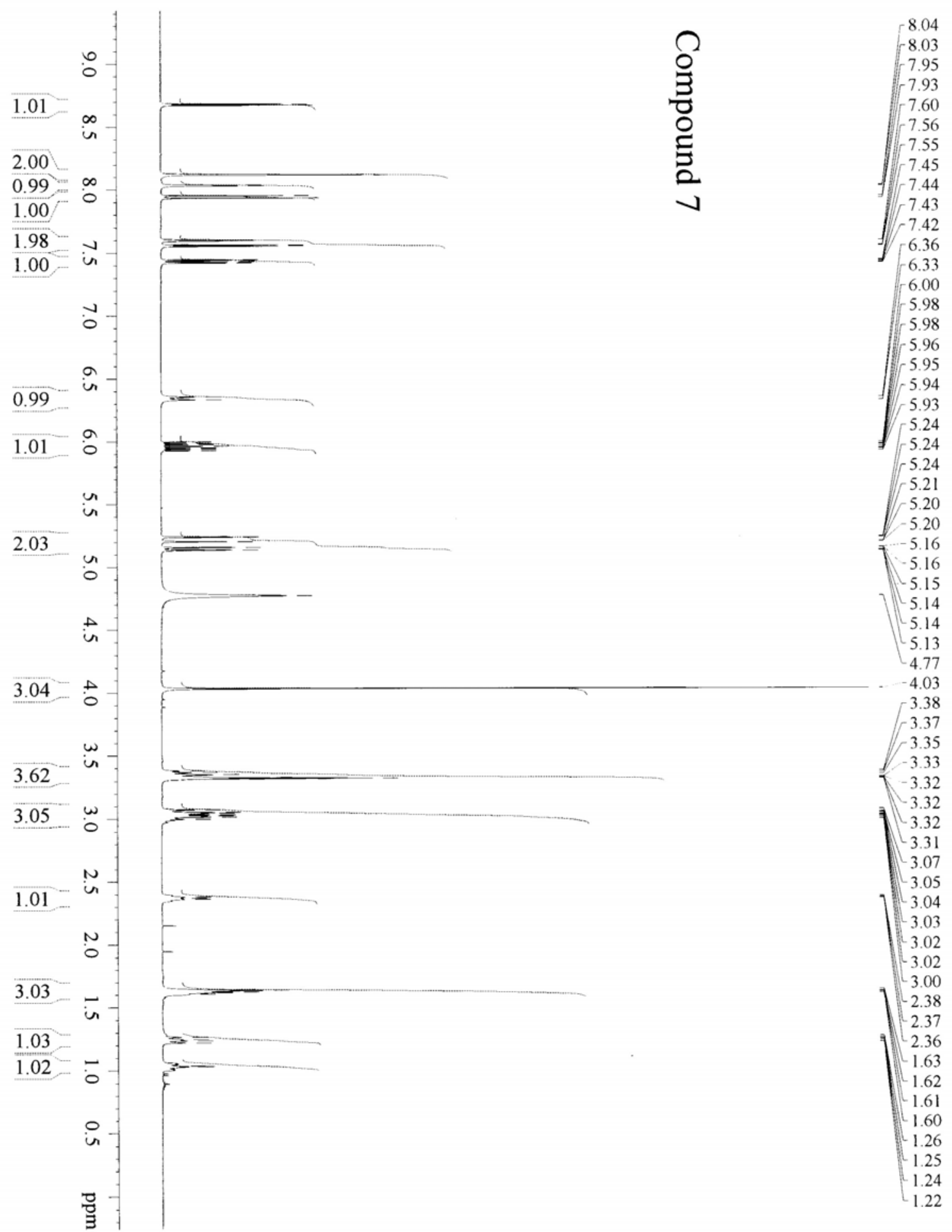

Figure 4. ${ }^{1} \mathrm{H}$ NMR spectra of 7 